\title{
Foil Calibration for IR Imaging Bolometer by Laser Irradiation*)
}

\author{
Ryuichi SANO, Byron J. PETERSON ${ }^{1)}$, Evgeny A. DRAPIKO ${ }^{1)}$, Dongcheol SEO ${ }^{2)}$, \\ Yuji YAMAUCHI ${ }^{3)}$ and Tomoaki $\mathrm{HINO}^{3)}$ \\ Graduate School of Engineering, Hokkaido University, Kita-13, Nishi-8, Kita-ku, Sapporo 060-8628, Japan \\ 1) National Institute for Fusion Science, 322-6 Oroshi-cho, Toki 509-5292, Japan \\ ${ }^{2)}$ National Fusion Research Institute, Daejeon 305-806, Republic of Korea \\ ${ }^{3)}$ Faculty of Engineering, Hokkaido University, Kita-13, Nishi-8, Kita-ku, Sapporo 060-8628, Japan
}

(Received 9 December 2011 / Accepted 4 March 2012)

\begin{abstract}
The IR imaging video bolometer (IRVB) provides the power distribution of plasma radiation. The radiation distribution is obtained from the temperature distribution on the bolometer foil. It is necessary to calibrate between the temperature distribution and the incident radiation power on the bolometer foil. This paper describes a new calibration technique for the foil which we have developed. The bolometer foil was irradiated with a He- $\mathrm{Ne}$ laser and the temperature distribution was measured by an IR camera while changing the irradiation position. The temperature distribution measured was analyzed by the comparison with the results calculated by FEM. We repeated this comparison while changing the parameters such as effective foil thickness and effective emissivity in the calculation until the calculated distribution converged to the measured one. The temperature distribution calculated by the FEM agreed well with the measured one, so the calibration between the radiation power and the temperature profile can be suitably conducted by this technique.
\end{abstract}

(c) 2012 The Japan Society of Plasma Science and Nuclear Fusion Research

Keywords: IRVB, plasma diagnostics

DOI: $10.1585 /$ pfr.7.2405039

\section{Introduction}

An IR imaging video bolometer (IRVB) [1] is a measurement instrument for plasma radiation. It is useful for the measurement of both radiation intensity and spatial distribution. These measurement data are useful for the operation of a fusion device. IRVBs have been used in LHD [2] and JT-60U [3] with a bolometer foil. An IRVB measures the plasma radiation by the temperature distribution on the bolometer foil which is observed by an IR camera. The bolometer foil was blackened by graphite spray to improve of the observation with an IR camera.

When the bolometer foil absorbs the radiation power, a 2D temperature distribution is formed on the foil and measured by an IR camera. The radiation power which is absorbed by the foil is given in terms of the temperature distribution on the foil by the following heat diffusion equations,

$$
\begin{aligned}
& -\Omega_{\mathrm{rad}}+\Omega_{\mathrm{bb}}+\frac{1}{\kappa} \frac{\partial T}{\partial t}=\frac{\partial^{2} T}{\partial x^{2}}+\frac{\partial^{2} T}{\partial y^{2}} \\
& \Omega_{\mathrm{rad}}=\frac{P_{\mathrm{rad}}}{k t_{\mathrm{f}}} \\
& \Omega_{\mathrm{bb}}=\frac{\varepsilon \sigma_{\mathrm{SB}}\left(T^{4}-T_{0}^{4}\right)}{k t_{\mathrm{f}}},
\end{aligned}
$$

where $T$ is the two-dimensional temperature distribution on the foil measured by the IR camera, $k$ the foil ther-

author's e-mail: doreiko@frontier.hokudai.ac.jp

*) This article is based on the presentation at the 21 st International Toki Conference (ITC21) mal conductivity, $t_{\mathrm{f}}$ the foil thickness, $\sigma_{\mathrm{SB}}$ the StefanBoltzmann constant, $\varepsilon$ the black body emissivity, $T_{0}$ the background temperature, $P_{\text {rad }}$ the incident radiation power density and $\kappa$ the thermal diffusivity of the foil. The plasma radiation distributions are calculated from the absorbed power with tomography techniques [4]. Then, the evaluation of the absorbed power is essential to estimate the radiation distributions. To obtain the absorbed power, we must evaluate the thermal characteristics distribution of the bolometer foil which are parameters in the heat diffusion equation.

The steady state temperature distribution depends on the distributions of foil parameter $k t_{\mathrm{f}}$ and $\varepsilon$ as shown in Eq. (1). These distributions are often non-uniform across the foil. So, the evaluations of the foil thickness and emissivity distribution are essential to calibrate the absorbed power from the data of the temperature distribution on the foil. The foil calibration techniques have been developed with a He-Ne laser [5] and ultra violet diode [6]. The calibration with the He-Ne laser is useful for obtaining the calibration data of steady state on the full face of foil. However, the calibration with a He-Ne laser can not be made for each position on the foil at which the laser was irradiated by neglecting the effect of the surrounding area and could not obtain the emissivity, so that an alternative technique for the calibration with consideration of the surrounding area is necessary. The purpose of this study is the development of a new technique to calibrate the power absorbed 
on the foil by the iteration method.

\section{Calibration Procedure}

\subsection{Calibration flow}

Figure 1 shows the calibration flow chart. This calibration has three steps. As a first step, we measured the bolometer foil temperature distribution under laser irradiation and fitted this distribution to a fitting function at all irradiation points. As a second step, we calculated temperature distribution using finite element method (FEM) by changing foil thickness and fitting the temperature distribution to the fitting function. We compared the calculated temperature distribution and the measured one to estimate the effective foil thickness at all irradiation points. As a third step, we carried out the same method as the second step but changing the foil emissivity to estimate the effective emissivity. We repeated the second and third steps with feedback to the FEM model until the FEM temperature distribution converged to the measured one.

\subsection{Experiment}

The evaluated foil was Pt. The foil was blackened by carbon spray on both sides The size of the foil was $90 \mathrm{~mm} \times 70 \mathrm{~mm}$. The nominal thickness was $2.5 \mu \mathrm{m}$. The foil mounted on a copper frame was installed in a vacuum chamber. The chamber was evacuated below $0.1 \mathrm{~Pa}$ to eliminate the heat conduction cooling by air.

We irradiated the bolometer foil with a He-Ne laser by changing the irradiation position by moving the foil in two dimensions, and then measured the 2D temperature distribution with an IR camera with a $\mathrm{ZnSe}$ IR window at room temperature. The camera was Indium Antimonide detector type, manufactured by FLIR with a wavelength of $3-5 \mu \mathrm{m}, 420 \mathrm{fps}, 320 \times 256$ pixels. The power of the He$\mathrm{Ne}$ laser was about $11.5 \mathrm{~mW}$ at the foil position. The spot diameter of the laser was about $0.6 \mathrm{~mm}$. Figure 2 shows the irradiation point on the foil. We divided the foil into to 63 parts with $10 \mathrm{~mm} \times 10 \mathrm{~mm}$ square. In this study, we have conducted the calibrations under the assumption that the thicknesses and emissivity of these squares were uniform in each square. The laser irradiation points were located on the center of each square. The blue crosses in Fig. 2 show the irradiation points and the red numbers show the irradiation point numbers.

In this study, the measured temperature distribution around the irradiated position was fitted to the distribution described in the following equation.

$$
T=T_{0}+\Delta T \exp \left(\frac{-\left(\frac{x}{w}\right)^{2 f a c}}{2}\right) .
$$

Figure 3 shows the typical results of the fitting. This fitting provided a peak size, $\Delta T$, a peak width, $w$, and a peak shape exponent, $f a c$, for each position. Using these fitting parameters, we conducted the following FEM analysis to calibrate the foil.

\section{Calibration flow chart}

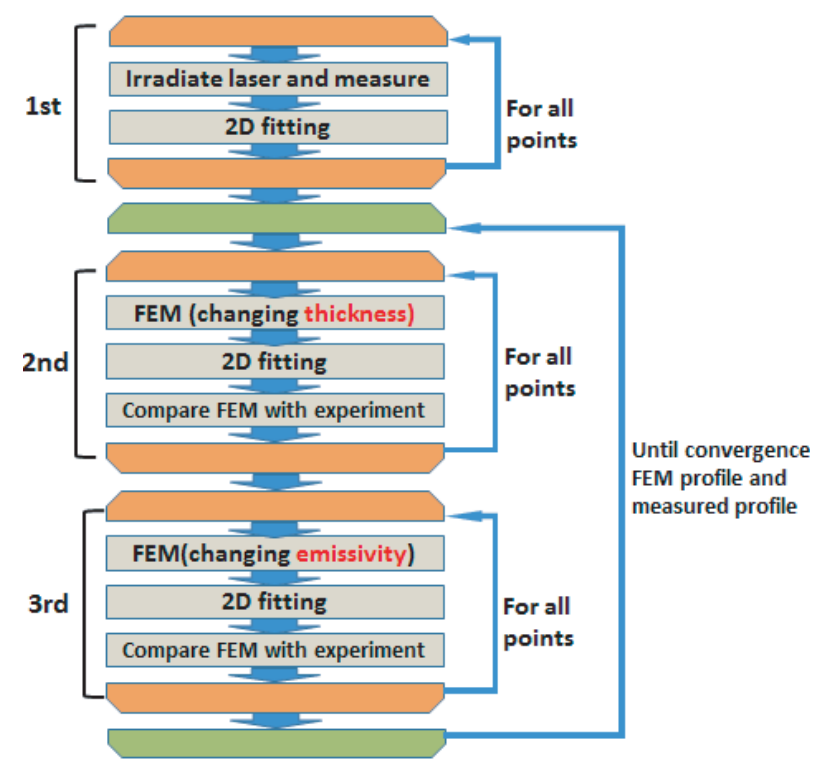

Fig. 1 Foil calibration flow chart.

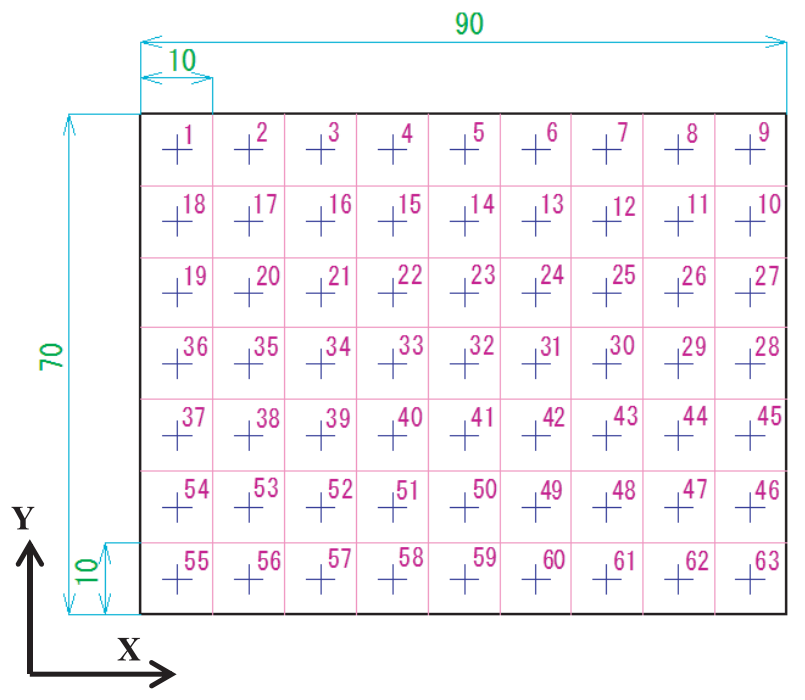

Fig. 2 Sections for calibration and laser-irradiation positions on foil.

\subsection{Foil calibration by FEM analysis}

We calibrated the Pt foil under laser irradiation at each point by FEM analysis with an FEM model which was sectioned as described in Fig. 2. These areas are not elements for the FEM analysis, the FEM analysis was performed with much smaller element than the ones described in Fig. 2, In this analysis, the foil thickness and the emissivity was changed individually for each section described in Fig. 2. In this FEM model, we set the temperature at foil edge to room temperature as boundary condition. We assumed the thermal conductivity, $k$, of the foil was that of pure Pt, 71.6 W/mK. We conducted the FEM analysis with the initial foil thickness of $2.5 \mu \mathrm{m}$ and initial emissivity of 0.91 for all areas. The foil temperature distribution was 
simulated using the initial emissivity with slight changes of the thicknesses.

The calculated temperature distributions were fit to Eq. (2) with the same peak shape power as each irradia-

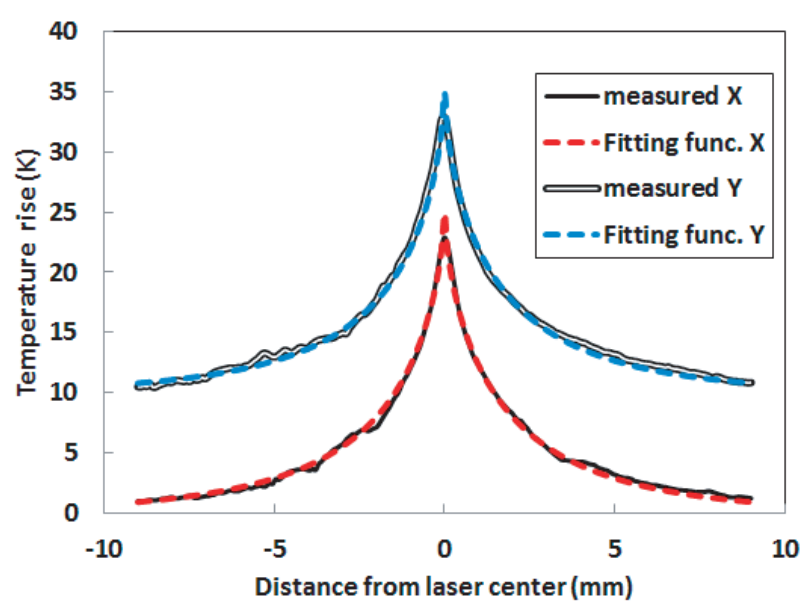

Fig. 3 Fitting between fitting function and the measured temperature. The temperature of $Y$ direction is shifted $10 \mathrm{~K}$ to upper.
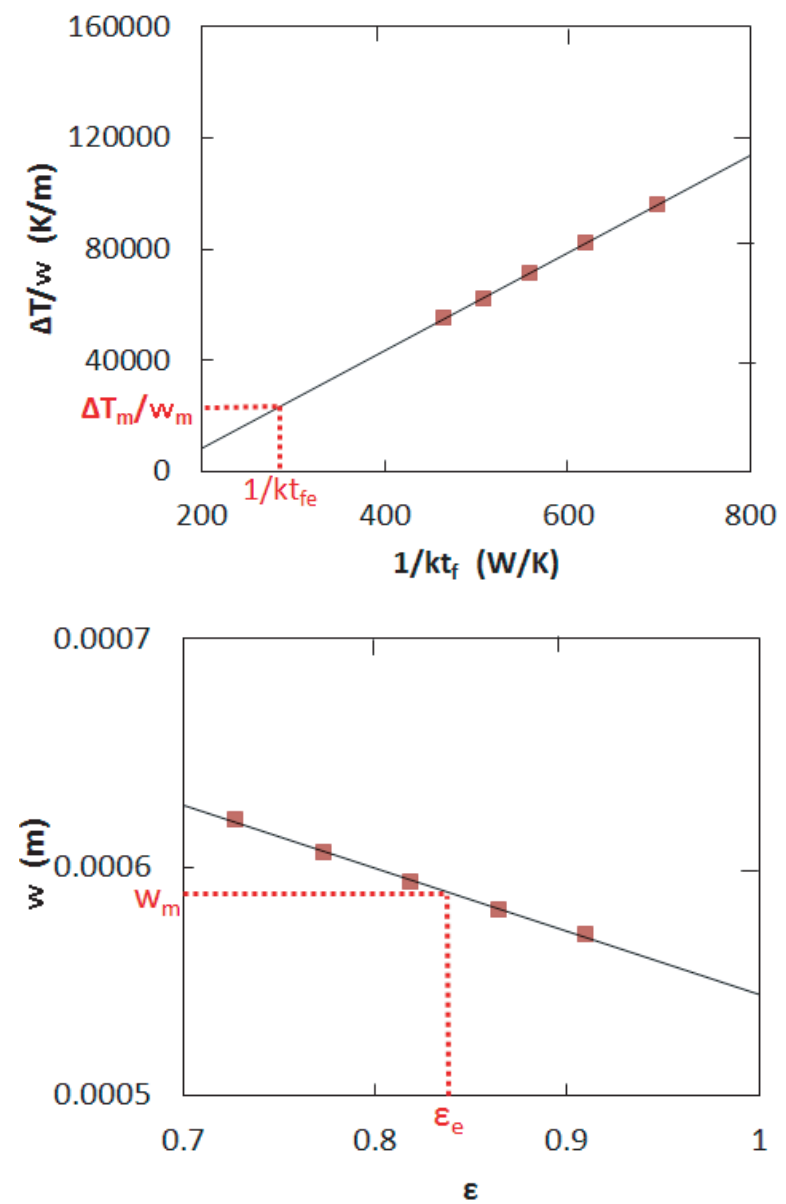

Fig. $4 \Delta T / w$ and $w$ at irradiation point 32 (foil center). $\Delta T_{\mathrm{m}}$ and $w_{\mathrm{m}}$ are measured value. $t_{\mathrm{fe}}$ and $\varepsilon_{\mathrm{e}}$ are estimated value. tion point of the experiment. The fitting provided $\Delta T$ and $w$ for the each calculated temperature distribution. The foil thickness at each position was estimated from these data by means of linear fitting in Fig. 3, which shows the relation of $\Delta T / w$ with $1 / k t_{\mathrm{f}}$. Next, the FEM analysis was conducted using the obtained thickness distribution with a slight change of emissivity. Figure 4 (b) shows the relation of $\varepsilon$ with peak width, $w$. We did linear fitting to this plot and projected the measured $w$ on the line to estimate its emissivity. The estimated emissivity was given to the FEM model.

We reiterate the FEM sequence described above, until the FEM temperature distribution suitably reproduced the measured one.

\section{Results and Discussions}

Figure 5 shows the 2D profile of the estimated thickness and emissivity. It is noted that the foil thickness and emissivity estimated by this analysis do not correspond to the nominal values because the FEM model did not take the influence of carbon coating and structural damage on emissivity and thermal conductivity into account. However, effective thermal response of the foil for the measurement of incident power distribution can be assessed with
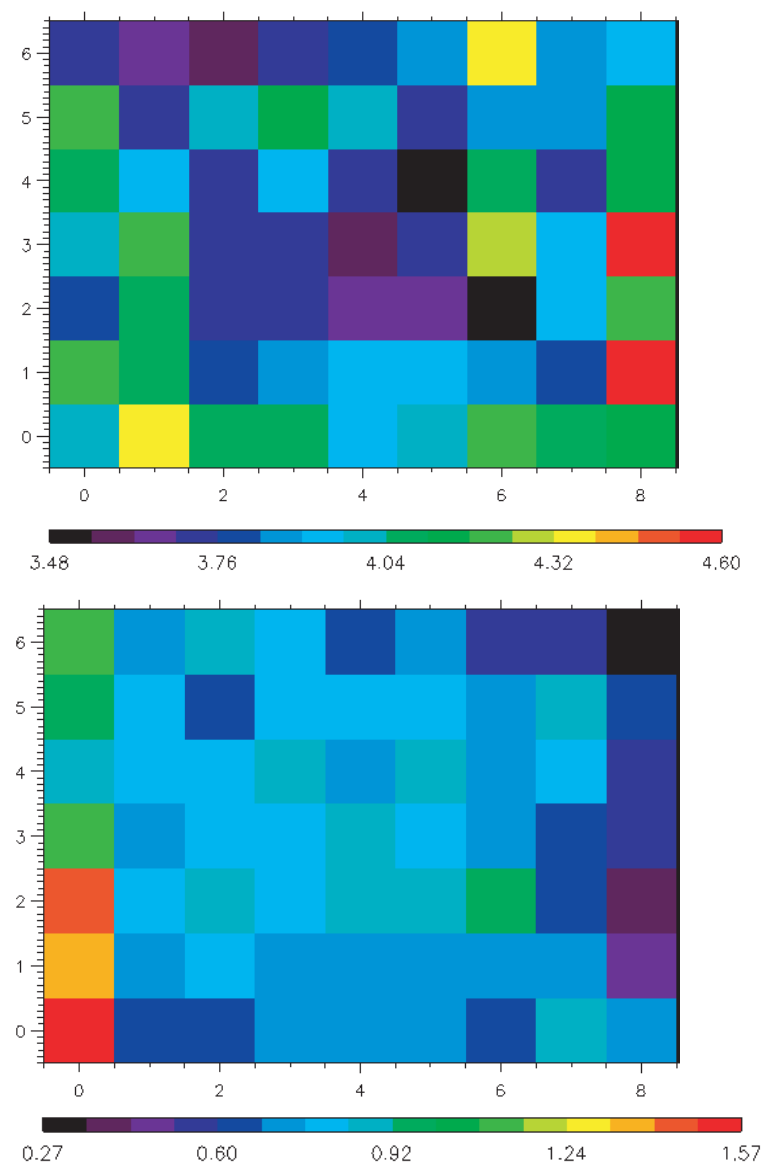

Fig. 5 The estimated foil thickness ( $\mu \mathrm{m})$ (Upper) and emissivity (Lower) from FEM analysis. 

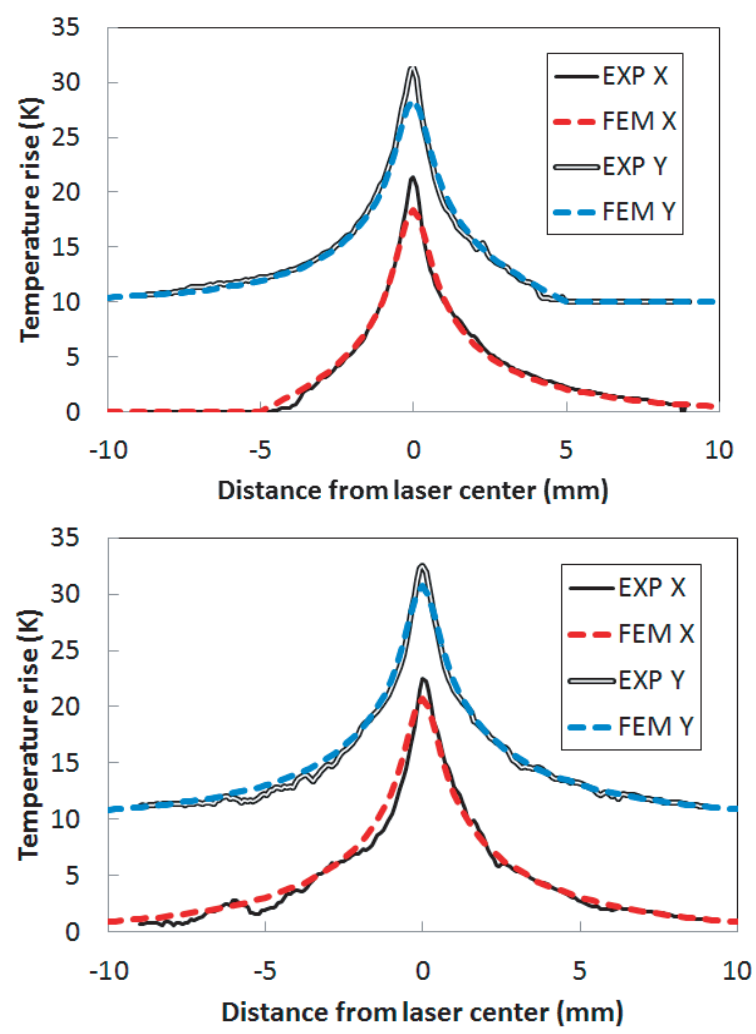

Fig. 6 Comparison between the FEM temperature distribution and measured at irradiation point 1 (Upper) and point 32 (lower). The temperature of $Y$ direction is shifted $10 \mathrm{~K}$ to upper.

the estimation.

Figure 6 shows comparisons between the measured temperature distribution and the one which were reproduced by using the parameters obtained by 4 iterations of FEM sequence for the center (position 32 in Fig. 2) and corner of the foil (position 1 in Fig. 2). The distribution estimated by the FEM sequences agreed well with the measured one except the top of the peaks. Namely, the measured temperature was higher than the estimated one at the top of the peak. This discrepancy would be attributed to difference of the adopted laser power density in the lase spot. This led to a discrepancy of heat flux distribution around the spot. The laser power density distribution were measured before previous experiment [5] and were adopted in the present study. The distribution might be changed in this experiment. However, the influence of the differences was limited only the estimation inside the laser spot.

These results indicate that the thermal response of the bolometer foil could be estimated by the present calibration. Therefore, the absorbed power could be obtained from the temperature distribution of the foil using the calibration data.

\section{Summary}

We developed a new calibration technique between radiation power and temperature distribution on the bolometer foil. The technique provided calibration data after four iterations. It is found that the FEM profile with this calibration data agreed well with the temperature profile measured. Therefore, this technique is useful to obtain calibration data for IRVB measurement.

\section{Acknowledgements}

The Authors would like to thank Mr. Hiromi Hayashi of NIFS for assistance with the FEM. This research was supported by NIFS LHD budget code NIFS11ULHH026 and NIFS collaboration budget code NIFS11KLEH015.

[1] B.J. Peterson, A.Yu. Kostrioukov, N. Ashikawa, M. Osakabe and S. Sudo, Rev. Sci. Instrum. 74(3), 2040 (2003).

[2] B.J. Peterson, S. Yoshimura, E.A. Drapiko, D.C. Seo, N. Ashikawa and J. Miyazawa, Fusion Sci. Technol. 58(1), 412 (2010).

[3] B.J. Peterson, S. Konoshima, H. Parchamy, M. Kaneko, T. Omori, D.C. Seo, N. Ashikawa, A. Sukegawa and JT-60U Team, J. Nucl. Mater. 363-365, 412 (2007).

[4] Y. Liu, N. Tamura, B.J. Peterson, N. Iwama, S. Konoshima, LHD Experimental Group and JT-60 Team, Plasma Fusion Res. 2, S1124 (2007).

[5] H. Parchamy, B.J. Peterson, S. Konoshima, H. Hayashi, D.C. Seo, N. Ashikawa and JT-60U Team, Rev. Sci. Instrum. 77, 10E515 (2006).

[6] E. Drapiko, B. Peterson, A. Alekseev and D.C. Seo, Rev. Sci. Instrum. 81, 10E116 (2010). 